\title{
Benzene Shape Photonic Crystal Fiber Based Plasma Sensor: Design and Analysis
}

\author{
Md. Toriqul ISLAM ${ }^{1}$, Md. Golam MOCTADER ${ }^{1 *}$, Kawsar AHMED $^{2,3}$, and \\ Sawrab CHOWDHURY ${ }^{3}$ \\ ${ }^{1}$ Department of Physics, Mawlana Bhashani Science and Technology University, Santosh, Tangail-1902, Bangladesh \\ ${ }^{2}$ Group of Bio-photomatix, Tangail-1902, Bangladesh \\ ${ }^{3}$ Department of Information and Communication Technology, Mawlana Bhashani Science and Technology University, \\ Santosh, Tangail-1902, Bangladesh \\ *Corresponding author: Md. Golam MOCTADER \\ E-mail: ronok12008@gmail.com, kawsar.ict@mbstu.ac.bd, and \\ k.ahmed.bd@ieee.org
}

\begin{abstract}
A novel benzene core photonic crystal fiber (BC-PCF) is proposed for plasma sensing applications. The proposed BC-PCF parameters have been tuned to gain high sensitivity, high numerical aperture (NA), and low confinement loss, and modality over the extensive variety of $0.7 \mu \mathrm{m}$ to $1.9 \mu \mathrm{m}$ wavelength. The explored results for the ideal structure have exhibited the high sensitivity up to $77.84 \%$ and negligible confinement loss of $7.9 \times 10^{-3} \mathrm{~dB} / \mathrm{m}$ at $1.3 \mu \mathrm{m}$ wavelength. The V-barometer remains under 2.405 over the whole working wavelength. So the proposed BC-PCF is a single mode fiber, which advances the long partition correspondence applications. Furthermore, high numerical aperture (NA) makes the fiber potential candidate in medical imaging applications. The plan of the sensor is to find out the creative potential outcomes in sensing applications.
\end{abstract}

Keywords: Optical sensor; relative sensitivity; numerical aperture; confinement loss; benzene core photonic crystal fiber (BC-PCF)

Citation: Md Toriqul ISLAM, Md. Golam MOCTADER, Kawsar AHMED, and Sawrab CHOWDHURY, "Benzene Shape Photonic Crystal Fiber Based Plasma Sensor: Design and Analysis," Photonic Sensors, 2018, 8(3): 263-269.

\section{Introduction}

Nowadays, looking for the scientific research it is detectable that logical research is going with optical fiber innovation by managing photonic crystal fiber (PCF). In contrast with regular optical fiber, PCFs are contributing to advancing optical detecting and in addition, optical correspondence by demonstrating some indistinguishable properties like additional guiding opportunity, low confinement loss, high sensitivity, high center power division, high numerical aperture (NA), vast powerful zone, high nonlinearity, high birefringence, and ultra-flattened dispersion [1-11]. Additionally, PCF likewise assumes a fundamental part in modern, biomedical, and natural detecting applications. Sensing can be communicated as the common activity amongst analysts and passing light through the fiber. PCF based sensors have turned out to be excessive mainstream by scientists because of its flexibility and adaptability. With the assistance of innovative headway, the optical sensors, for example, gas sensor, compound sensor, temperature sensor, pressure sensor, biosensor, twist sensor, strain sensor,

Received: 17 February 2018 / Revised: 29 May 2018

(C) The Author(s) 2018. This article is published with open access at Springerlink.com

DOI: $10.1007 / \mathrm{s} 13320-018-0495-8$

Article type: Regular 
refractive index sensor, vibration sensor, DNA sensor, protein sensor, dampness sensor, and $\mathrm{pH}$ sensor are refreshing quickly [12-23]. Be that as it may, outlining an adaptable and dependable sensor is a testing issue because of the absence of proficient detecting and additionally other directing properties.

Last few decades ago, to get scrumptious optical properties, different geometric shapes, for example, hexagonal, octagonal, circular, decagonal, porous, spiral, and hybrid cladding, were presented. In 2016, Ahmed et al. [24] proposed hexagonal, square, and octagonal shape PCFs for sensing applications. At $1.33 \mu \mathrm{m}$ wavelength, the octagonal PCF increased most extreme sensitivity of $46.87 \%$ for ethanol analyte. But they did not mention any other parameters without sensitivity and confinement loss. The same authors [25] proposed a micro structure octagonal PCF for liquid sensing applications and improved the sensitivity of $47.35 \%$ and confinement loss of $2.40 \times 10^{-4} \mathrm{~dB} / \mathrm{m}$ at $1.33 \mu \mathrm{m}$ wavelength.

In 2016, Asaduzzaman et al. [26] enhanced the sensitivity up to $49.17 \%$ and confinement loss of $2.75 \times 10^{-10} \mathrm{~dB} / \mathrm{m}$ by presenting hybrid cladding PCF. The authors did not specify the modality and NA properties with the exception of sensitivity and confinement loss. In last 2016, Paul et al. [27] enhanced the sensitivity up to $64.19 \%$ for S-band by proposing folded cladding PCF. In addition, they showed and picked up the NA of 0.36 for U-band. In 2017, same authors enhanced the sensitivity of 1.06 times for identification liquor from drinks [28]. However, they didn't demonstrate the other pivotal parameters. Moreover, still now there is no published articles for the PCF based plasma sensor. So in light of the past writing articles, there is still gigantic space to outline plasma sensors to accomplish better guiding properties.

In this article, a circular-cladding and benzene-core based PCF has been proposed for proficient conveyance of sensing applications. The research has uncovered the extreme sensitivity and confinement loss of $77.23 \%$ and $5.15 \times 10^{-3} \mathrm{~dB} / \mathrm{m}$, respectively. In addition, the ideal structure has likewise picked up the high NA. The proposed fiber is applicable in detecting plasma as well as biomedical imaging applications.

\section{Geometries of the proposed P-HPCF}

The end-faced view of the proposed benzene core photonic crystal fiber (BC-PCF) is presented in Fig. 1. At BC-PCF, the microstructure benzene shaped core is infiltrated with plasma $(n=1.350)$. The circular five layers air hole surrounded by the core acts as a cladding region. The core region has three-layer-microstructure air holes where the radius of the core is homogenous except 6 corner air holes. The microstructure core with benzene shape contains 6 air holes in a benzene manner. The diameters of the air holes in the core region are denoted by $D_{\text {co }}$, and the distance between holes (pitch) is denoted by $\Lambda_{\mathrm{co}}$. The diameter of 6 corner air holes is $0.8 \mu \mathrm{m}$ where the other air holes' diameter is $0.3 \mu \mathrm{m}$. The cladding is arranged with five layers of the air holes which remain the same with the structures different in the core region. The air filling ratio (AFF) is a very critical factor in the fabrication process. In the proposed BC-PCF, the AFF remains below $90 \%$ both for the core and cladding regions. So the proposed $\mathrm{BC}-\mathrm{PCF}$ can be easily fabricated using any well-known techniques.

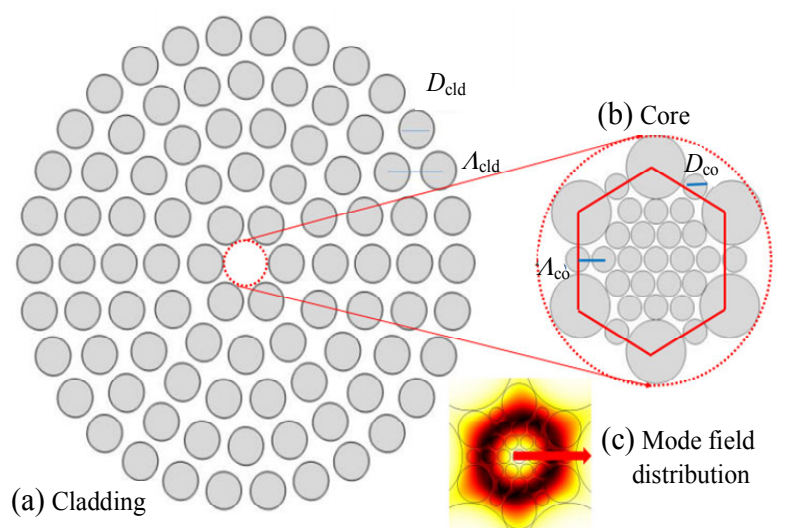

Fig. 1 Schematic of end-faced view of proposed BC-PCF: (a) cladding region, (b) core, and (c) modal intensity.

The diameters of the air holes at cladding are denoted by $D_{\text {cld, }}$, and the pitch of the two adjacent air holes between the two different layers of air holes is denoted by $\Lambda_{\text {cld. }}$. Silica is used as hosting material for 
the better optical guiding properties for a wider wavelength range [29]. The COMSOL multi-physics 4.2 software has been employed to simulate and design the proposed structure. An outer boundary known as perfectly matched layer (PML) has been applied for diminished nonphysical scattering with a thickness of $10 \%$ following the articles [30-33]. Figure 1(c) visualizes the modal intensity of the proposed structure and it is nicely exhibited that light is tightly confined through the core region.

\section{Synopsis of numerical method}

Finite element method (FEM) is used for analyzing our proposed PCF structure which has been utilized for the electromagnetic simulation of the proposed chemical (Plasma) sensor PCF. We consider two very important parameters, sensitivity and confinement loss, which are very closely related to our PCF structure. We should solve the Maxwell equation by utilizing the circular perfectly matched boundary layer (PML). All numerical calculations have been done following the previously published articles [34].

The host material of the proposed PCF is pure silica. Silica has a refractive index dependency on wavelength followed by Sellmeier equation as

$$
n(\lambda)=\sqrt{1+\frac{B_{1} \lambda^{2}}{\lambda^{2}-C_{1}}+\frac{B_{2} \lambda^{2}}{\lambda^{2}-C_{2}}+\frac{B_{3} \lambda^{2}}{\lambda^{2}-C_{3}}}
$$

where $B_{1}=0.6961663, B_{2}=0.4079426, B_{3}=$ $0.8974794, C_{1}=0.0684043, C_{2}=0.1162414, C_{3}=$ 98.96161, and $n(\lambda)$ is the refractive index for corresponding operating wavelength $\lambda$ in $\mu \mathrm{m}$.

A powerful numerical simulation tool is the full vectorial finite-element method. Various photonic waveguide devices are numerically analyzed by FEM. FEM processes complex structure PCF in homogenous subspaces and then is computed with Maxwell's vector equation as

$$
\nabla \times\left([\mathbf{S}]^{-1} \nabla \times \mathbf{E}\right)-k_{0}^{2} n^{2}[\mathbf{S}] \mathbf{E}=0
$$

where $[\mathbf{S}]$ is the PML matrix including PML parameters $S_{x}$ and $S_{y}, \mathbf{E}$ is the electric field vector, and the wave number is $k_{0}$ which is a function of wavelength $(\lambda)$ followed as follows:

$$
[\mathbf{S}]=\left[\begin{array}{ccc}
\frac{S_{y}}{S_{x}} & 0 & 0 \\
0 & \frac{S_{y}}{S_{x}} & 0 \\
0 & 0 & S_{y} S_{x} \\
K_{0}=\frac{2 \pi}{\lambda} .
\end{array}\right]
$$

During the simulations time, COMSOL solves the Maxwell's equation internally, and simulation outcomes provide the propagation constant of the operating wavelength. The real part produces the effective mode index, the imaginary part produces the confinement loss, and they are calculated as follows:

$$
n_{\text {eff }}=R_{e} \times \frac{\beta}{K_{0}} .
$$

When the power flows through the fiber, some power penetrates into cladding region of the fiber, which causes confinement loss or leakage loss as follows:

$$
L_{c}\left[\frac{\mathrm{dB}}{\mathrm{m}}\right]=8.686 K_{0} I_{m}\left(n_{\mathrm{eff}}\right) \times 10^{6} .
$$

The effective mode area of the proposed PCF structure can be calculated by the propagating mode area as follows:

$$
A_{\text {eff }}=\frac{\left(\iint|E(x, y)|^{2} d x d y\right)^{2}}{\iint|E(x, y)|^{4} d x d y} .
$$

The NA of the PCF can be calculated as follows:

$$
N A \approx\left(1+\frac{n A_{\text {eff }}}{\lambda^{2}}\right)^{-\frac{1}{2}} .
$$

\section{Numerical results and discussion}

The convergence error of any PCF is the defective modes in PCF with compactly supported perturbations. The convergence error of the proposed BC-PCF is very low. The value of convergence error is $5.28 \times 10^{6}$. To get greater sensitivity, the core region and cladding region of the proposed structure are tuned by changing values 
of cladding diameter, cladding pitch, core diameter, and core pitch setting on the benzene shape core. In Fig. 2, the sensitivity is visualized as a function of wavelength for different cladding pitches by keeping all the parameters constant. It is nicely exhibited that there is no much variation of sensitivity for cladding pitch variation. From the graph, the optimum sensitivity of $73.25 \%$ is found at the wavelength of $1.3 \mu \mathrm{m}$.

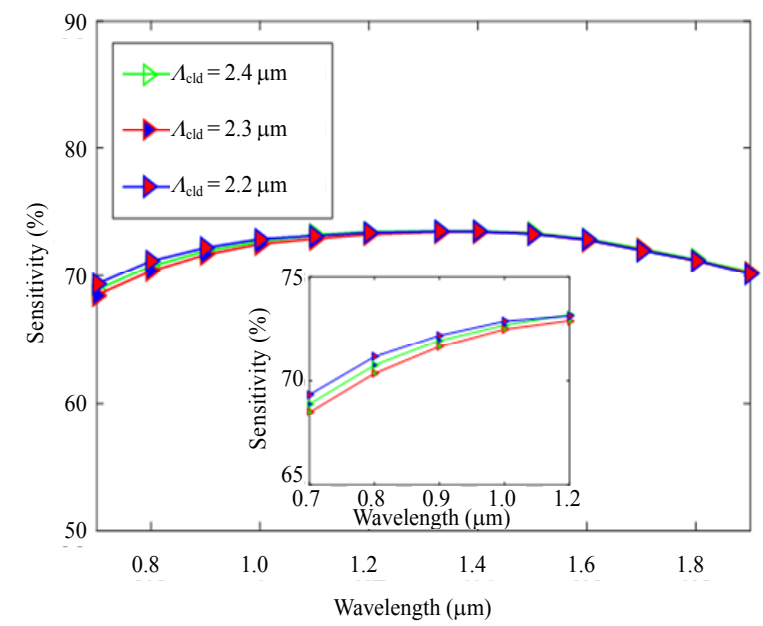

Fig. 2 Variation of sensitivity verses applied wavelength due to tuning the cladding pitch when other parameters are kept constant.

In Fig. 3, the changing rate of sensitivity has been revealed by tuning cladding diameter. The optimum sensitivity of $73.25 \%$ is found at the wavelength of $1.3 \mu \mathrm{m}$. Moreover, the higher air holes diameter enhances the sensitivity. These outcomes are high noticeable than that reported in [35].

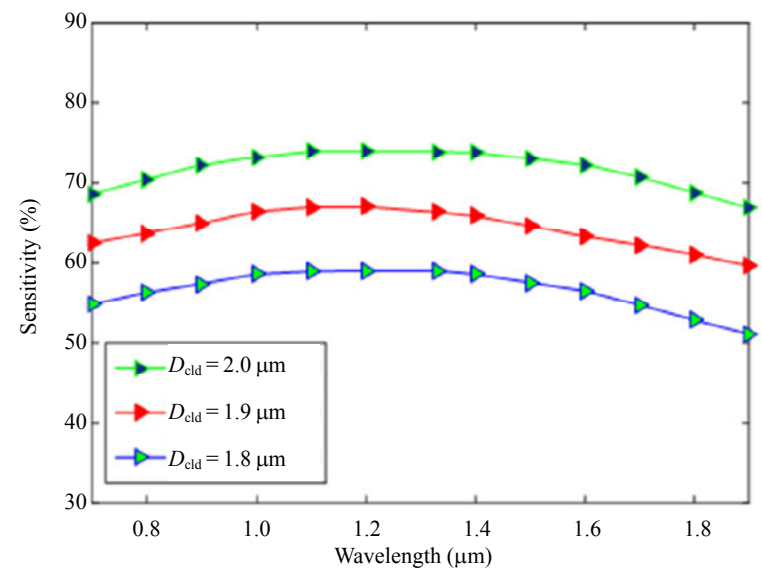

Fig. 3 Variation of sensitivity verses applied wavelength due to tuning the cladding diameter when other parameters are kept constant.
In Fig. 4, the sensitivity is pictured as a component of wavelength for various core diameters to keep every parameter steady. It is shown that there is a variety of sensitivities for cladding pitch. From the diagram, the ideal sensitivity of $77.96 \%$ is found at the wavelength of $1.3 \mu \mathrm{m}$. It is clearly pictured that the increment of core air holes diameter enhances the sensitivity. This outcome is exceedingly identical with that of the beforehand distributed articles [36-38]. The changing rate of sensitivity has been uncovered by tuning core pitch which is demonstrated in Fig. 5. The ideal sensitivity of $76.32 \%$ is found at the wavelength of $1.3 \mu \mathrm{m}$. In addition, the sensitivity is enhanced by the lower value of pitch. These results are highly observable than those reported in previous articles [39-41].

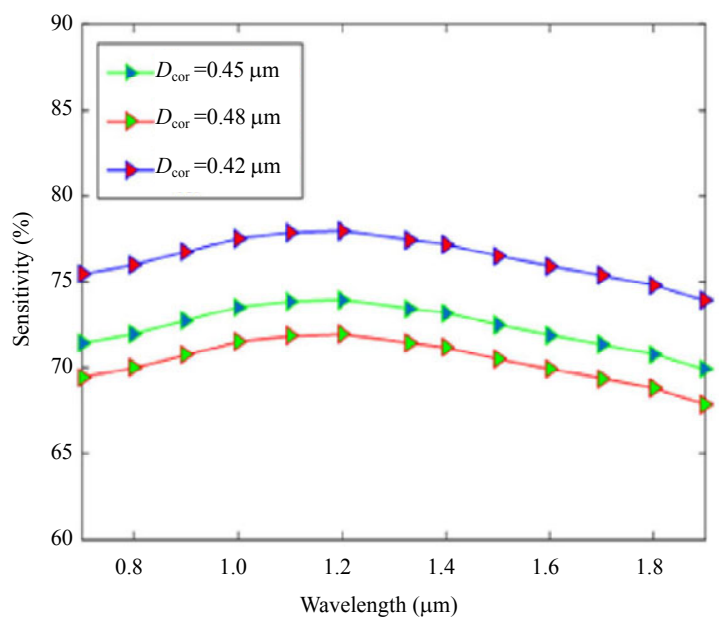

Fig. 4 Variation of sensitivity verses applied wavelength due to tuning the core diameter when other parameters are kept constant.

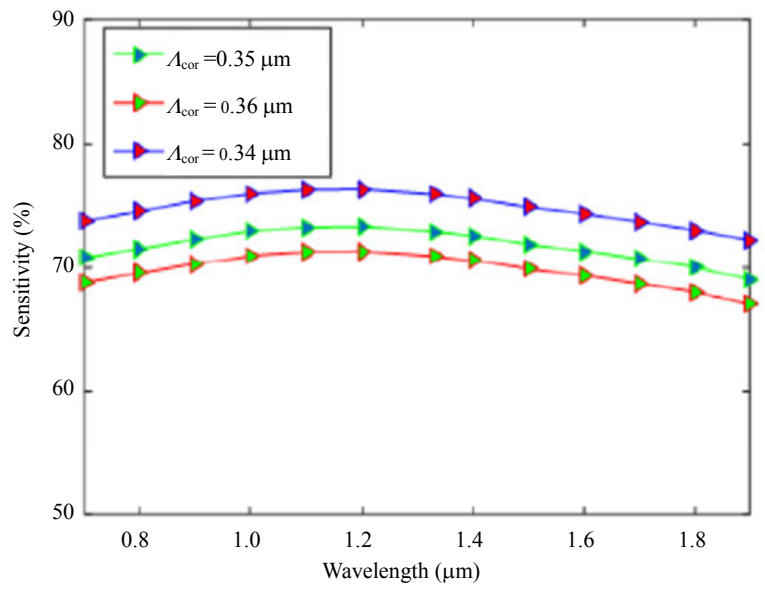

Fig. 5 Variation of sensitivity verses applied wavelength due to tuning the core pitch when other parameters are kept constant. 
From Fig. 1(c), it is noticeable that the light is well confined into the core region, so the leakage loss will be very low. The lower confinement loss of the proposed BC-PCF is shown in Fig. 6.

Powerful zone and nonlinearity decencies of photonic precious crystal fiber are driving the optical gadgets. In the correspondence territory, the information movement is expanding step by step. Subsequently, PCF with vast powerful territory is required if there should be an occurrence of high information movement transmission. Now the modality of any fiber can be checked by using (9). In Fig. 7, V-parameter stays under 2.405 over the entire working wavelength range. So the proposed PCF is a single mode fiber, which advances the long separation correspondence applications.

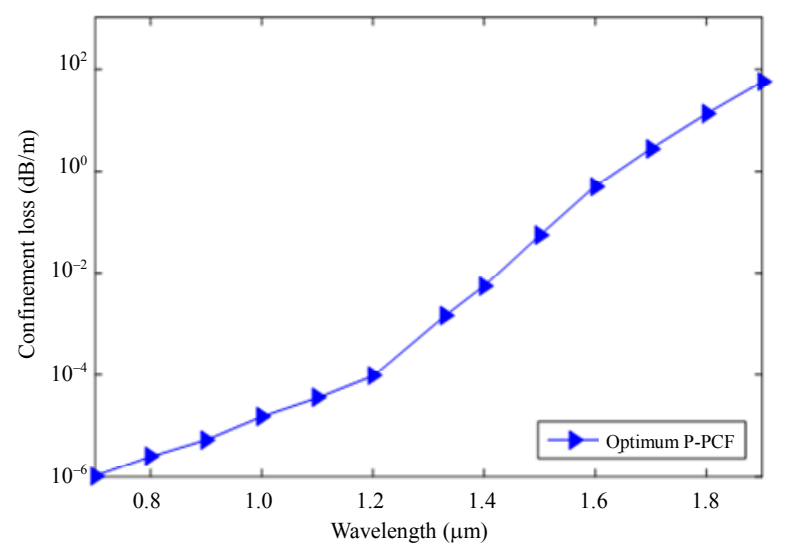

Fig. 6 Variation of confinement loss verses applied wavelength for the optimum structure.

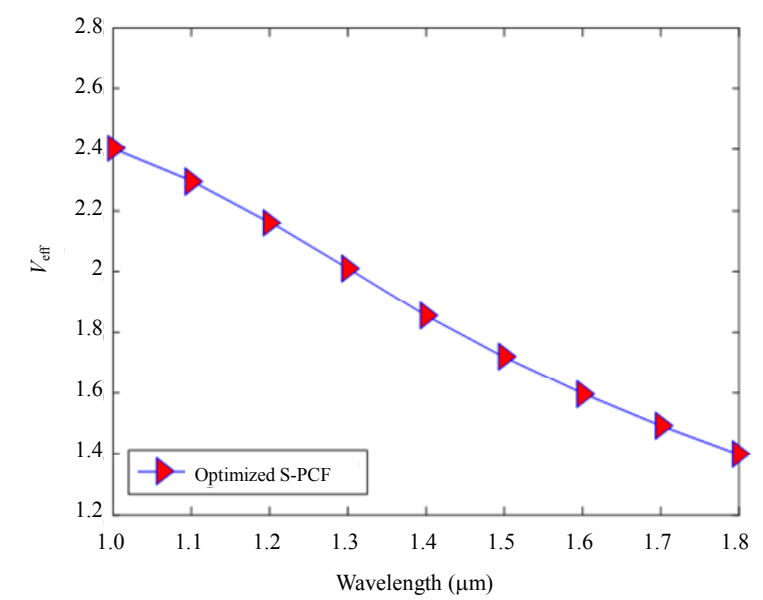

Fig. $7 V_{\text {eff }}$ as a function of wavelength for the optimum structure.

Tightly confinement of light into the core region introduces a smaller effective area and higher NA.
But it is noted that for silica based PCF the value of NA more than 0.4 is uncommon [42]. Figure 8 demonstrates the highest NA of 0.58 which makes the fiber a potential candidate in biomedical imaging applications. This value is highly noticeable than those in the previously reported articles [43].

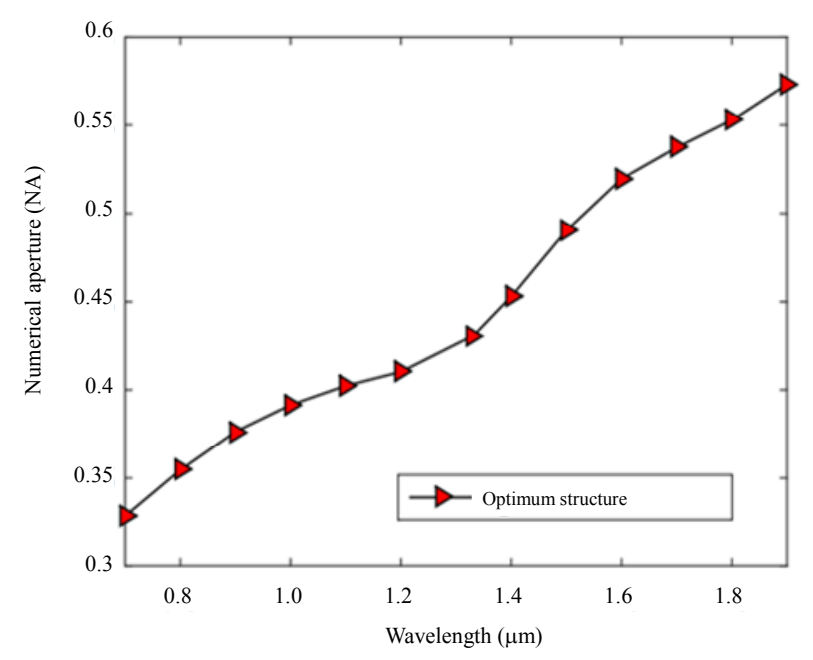

Fig. 8 NA as a function of wavelength for the optimum structure.

Table 1 compares the simulation results in research papers. It is clearly visualized that compared with other papers, the sensitivity obtained in our paper is higher and the concentration loss is lower.

Table 1 Comparison of simulated results in different previous papers for chemical sensing at $1.33 \mu \mathrm{m}$ wavelength.

\begin{tabular}{cccc}
\hline Articles & Sensitivity (\%) & Concentration & Published year \\
\hline Ref. [36] & 23.05 & $5.74 \times 10^{-6}$ & 2017 \\
Ref. [43] & 52.66 & $1.41 \times 10^{-10}$ & 2017 \\
Ref. [42] & 55.10 & $7.23 \times 10^{-3}$ & 2017 \\
Ref. [39] & 49.17 & $2.75 \times 10^{-10}$ & 2018 \\
This paper & 77.84 & $7.90 \times 10^{-3}$ & 2018 \\
\hline
\end{tabular}

\section{Conclusions}

A novel benzene core photonic crystal fiber (BC-PCF) based plasma sensor has been introduced, and different guiding properties are pursued. After theoretical modeling and numerical simulation using COMSOL Multi Physics 4.2 version, high sensitivity of $77.84 \%$ and low confinement loss of $7.9 \times 10^{-3} \mathrm{~dB} / \mathrm{m}$ at $1.3 \mu \mathrm{m}$ are obtained. In addition, the proposed $\mathrm{BC}-\mathrm{PCF}$ plasma sensor is realizable by using any well-known fabrication technique. Furthermore, the maximum NA of 0.58 is obtained 
at $1.9 \mu \mathrm{m}$ wavelength which makes the fiber an essential candidate in medical imaging applications. For the whole operating wavelength, the proposed BC-PCF also behaves a single mode fiber and makes the fiber potential fiber in long-distance applications.

Open Access This article is distributed under the terms of the Creative Commons Attribution 4.0 International License (http://creativecommons.org/licenses/by/4.0/), which permits unrestricted use, distribution, and reproduction in any medium, provided you give appropriate credit to the original author(s) and the source, provide a link to the Creative Commons license, and indicate if changes were made.

\section{References}

[1] X. Sang, P. L. Chu, and C. Yu, "Applications of nonlinear effects in highly nonlinear photonic crystal fiber to optical communications," Optical and Quantum Electronics, 2005, 37(10): 965-994.

[2] K. P. Hansen, "Introduction to nonlinear photonic crystal fibers," Journal of Optical and Fiber Communications Reports, 2005, 2(3): 226-254.

[3] A. Ortigosa-Blanch, J. C. Knight, W. J. Wadsworth, J. Arriaga, B. J. Mangan, T. A. Birks, et al., "Highly birefringent photonic crystal fibers," Optics Letters, 2000, 25(18): 1325-1327.

[4] T. P. Hansen, J. Broeng, S. E. Libori, E. Knudsen, A. Bjarklev, J. R. Jensen, et al., "Highly birefringentindex-guiding photonic crystal fibers," IEEE Photonics Technology Letters, 2001, 13(6): 588-590.

[5] G. W. An, S. G. Li, X. Yan, X. N. Zhang, Z. Y. Yuan, and Y. N. Zhang, "High-sensitivity and tunable refractive index sensor based on dual-core photonic crystal fiber," Journal of the Optical Society of America B, 2016, 33(7): 1330-1334.

[6] W. Qian, C. L. Zhao, S. He, X. Dong, S. Zhang, Z. Zhang, et al., "High-sensitivity temperature sensor based on an alcohol-filled photonic crystal fiber loop mirror," Optics Letters, 2011, 36(9): 1548-1550.

[7] S. Olyaee and F. Taghipour, "Ultra-flattened dispersion hexagonal photonic crystal fiber with low confinement loss and large effective area," IET Optoelectronics, 2012, 6(2): 82-87.

[8] A. Yin and L. Xiong, "Highly nonlinear with low confinement losses square photonic crystal fiber based on a four-hole unit," Infrared Physics \& Technology, 2014, 66(9): 29-33.

[9] K. Kishor, R. K. Sinha, and A. D. Varshney,
"Experimental verification of improved effective index method for endlessly single mode photonic crystal fiber," Optics and Lasers in Engineering, 2012, 50(2): 182-186.

[10] H. Ademgil and S. Haxha, "Endlessly single mode photonic crystal fiber with improved effective mode area," Optics Communications, 2012, 285(6): 1514-1518.

[11] T. A. Birks, J. C. Knight, and P. S. J. Russell, "Endlessly single-mode photonic crystal fiber," Optics Letters, 1997, 22(13): 961-963.

[12] Y. H. Chang, Y. Y. Jhu, and C. J. Wu, "Temperature dependence of defect mode in a defective photonic crystal," Optics Communications, 2012, 285(6): 1501-1504.

[13] Y. Liu and H. W. M. Salemink, "All-optical on-chip sensor for high refractive index sensing in photonic crystals," Europhysics Letters, 2014, 107(3): 1160-1170.

[14] S. Z. Zheng, Y. N. Zhu, and S. Krishnaswamy, "Nanofilm-coated photonic crystal fiber long-period gratings with modal transition for high chemical sensitivity and selectivity," SPIE, 2012, 8346(14): 1844-1864.

[15] C. K. Lee and J. Thillaigovindan, "Optical nanomechanical sensor using a silicon photonic crystal cantilever embedded with a nanocavity resonator," Applied Optics, 2009, 48(10): 1797-1803.

[16] S. Olyaee and A. A. Dehghani, "Ultrasensitive pressure sensor based on point defect resonant cavity in photonic crystal," Sensor Letters, 2013, 11(10): 1854-1859.

[17] Y. N. Zhang, Y. Zhao, and Q. Wang, "Multi-component gas sensing based on slotted photonic crystal waveguide with liquid infiltration," Sensors and Actuators B: Chemical, 2013, 184(8): 179-188.

[18] M. Morshed, M. F. H. Arif, S. Asaduzzaman, and K. Ahmed, "Design and characterization of photonic crystal fiber for sensing applications," European Scientific Journal, 2015, 11(12): 228-235.

[19] T. W. Lu and P. T. Lee, "Ultra-high sensitivity optical stress sensor based on double-layered photonic crystal microcavity," Optics Express, 2009, 17(3): 1518-1526.

[20] P. Hu, X. Dong, W. C. Wong, L. H. Chen, K. Ni, and C. C. Chan, "Photonic crystal fiber interferometric $\mathrm{pH}$ sensor based on polyvinyl alcohol/polyacrylic acid hydrogel coating," Applied Optics, 2015, 54(10): 2647-2652.

[21] W. C. Lai, S. Chakravarty, Y. Zou, and R. T. Chen, "Multiplexed detection of xylene and trichloroethylene in water by photonic crystal absorption spectroscopy," Optics Letters, 2013, 
38(19): 3799-3802.

[22] E. K. Akowuah, T. Gorman, H. Ademgil, S. Haxha, G. K. Robinson, and J. V. Oliver, "Numerical analysis of a photonic crystal fiber for biosensing applications," IEEE Journal of Quantum Electronics, 2012, 48(11): 1403-1410.

[23] M. B. Pushkarsky, M. E. Webber, O. Baghdassarian, L. R. Narasimhan, and C. K. N. Patel, "Laser-based photoacoustic ammonia sensors for industrial applications," Applied Physics B, 2002, 75(2-3): 391-396.

[24] K. Ahmed and M. Morshed, "Design and numerical analysis of microstructured-core octagonal photonic crystal fiber for sensing applications," Sensing and Biosensing Research, 2016, 7: 1-6.

[25] K. Ahmed, M. Morshed, S. Asaduzzaman, and M. F. H. Arif, "Optimization and enhancement of liquid analyte sensing performance based on square-cored octagonal photonic crystal fiber," OptikInternational Journal for Light and Electron Optics, 2017, 131: 687-696.

[26] S. Asaduzzaman, K. Ahmed, T. Bhuiyan, and T. Farah, "Hybrid photonic crystal fiber in chemical sensing," Springerplus, 2016, 5(1): 1-11.

[27] B. K. Paul, K. Ahmed, S. Asaduzzaman, and M. S. Islam, "Folded cladding porous shaped photonic crystal fiber with high sensitivity in optical sensing applications: design and analysis," Sensing and Biosensing Research, 2017, 12: 36-42.

[28] B. K. Paul, M. S. Islam, K. Ahmed, and S. Asaduzzaman, "Alcohol sensing over $\mathrm{O}+\mathrm{E}+\mathrm{S}+\mathrm{C}+$ $\mathrm{L}+\mathrm{U}$ transmission band based on porous cored octagonal photonic crystal fiber," Photonics Sensors, 2017, 7(2): 123-130.

[29] K. Ahmed, I. Islam, B. K. Paul, S. Islam, S. Sen, S. Chowdhury, et al., "Effect of photonic crystal fiber background materials in sensing and communication applications," Materials Discovery, 2017, 7: 8-14.

[30] M. S. Islam, B. K. Paul, K. Ahmed, S. Asaduzzaman, M. I. Islam, S. Chowdhury, et al., "Liquid-infiltrated photonic crystal fiber for sensing purpose: design and analysis," Alexandria Engineering Journal, 2017: 1-8.

[31] K. Ahmed and M. Morshed, "Design and numerical analysis of microstructured-core octagonal photonic crystal fiber for sensing applications," Sensing and Biosensing Research, 2016, 7: 1-6.

[32] B. K. Paul, M. S. Islam, S. Chowdhury, S. Asaduzzaman, and K. Ahmed, "Porous core photonic crystal fiber based chemical sensor," in Proceeding of IEEE 9th International Conference on Electrical and Computer Engineering (ICECE), Dhaka, Bangladesh, 2016, pp. 251-254.

[33] S. Sen, S. Chowdhury, K. Ahmed, and S. Asaduzzaman, "Design of a porous cored hexagonal photonic crystal fiber based optical sensor with high relative sensitivity for lower operating wavelength," Photonic Sensors, 2017, 7(1): 55-65.

[34] S. Chowdhury, K. Ahmed, S. Sen, and S. Asaduzzaman, "Design of highly sensible porous shaped photonic crystal fiber with strong confinement field for optical sensing," Optik-International Journal for Light and Electron Optics, 2017, 142: 541-549.

[35] A. Islam, M. Shadidul, B. K. Paul, K. Ahmed, S. Asaduzzaman, M. I. Islam, et al., "Liquid-infiltrated photonic crystal fiber for sensing purpose: design and analysis," Alexandria Engineering Journal, 2017: 1-8.

[36] M. H. Kabir, M. B. A. Miah, S. Asaduzzaman, and K. Ahmed, "Slotted corecircular PCF in chemical sensing applications," Ukrainian Journal of Physics, 2017, 62(7): 589-593.

[37] S. Asaduzzaman, K. Ahmed, M. F. H. Arif, and M. Morshed, "Application of microarray-core based modified photonic crystal fiber in chemical sensing," in Proceeding of IEEE Conference in Electrical and Electronics Engineering, Rajshahi, Bangladesh, 2015, pp. 41-44.

[38] S. Asaduzzaman, K. Ahmed, M. F. H. Arif, and M. Morshed, "Proposal of a simple structure Photonic crystal fiber for lower indexed Chemical sensing," in Proceeding of Computer and Information Technology, Dhaka, Bangladesh, 2015: 127-131.

[39] M. S. Islam, J. Sultana, K. Ahmed, M. R. Islam, A. Dinovitser, B. W. Ng, et al., "A novel approach for spectroscopic chemical identification using photonic crystal fiber in the terahertz regime," IEEE Sensors Journal, 2018, 18(2): 575-582.

[40] M. F. H. Arif, M. J. H. Biddut, K. Ahmed, and S. Asaduzzaman, "Simulation based analysis of formalin detection through photonic crystal fiber," in Proceeding of Electronics and Vision (ICIEV), Dhaka, Bangladesh, 2016, pp. 776-779.

[41] M. F. H. Arif, S. Asaduzzaman, K. Ahmed, and M. Morshed, "High sensitive PCF based chemical sensor for ethanol detection," in Proceeding of Electronics and Vision (ICIEV), Dhaka, Bangladesh, 2016, pp. 6-9.

[42] I. Islam, K. Ahmed, S. Asaduzzaman, B. K. Paul, T. Bhuiyan, S. Sen, et al., "Design of single mode spiral photonic crystal fiber for gas sensing applications," Sensing and Bio-Sensing Research, 2017, 13: 55-62.

[43] S. Chowdhury, S. Sen, K. Ahmed, B. K. Paul, M. B. A. Miah, S. Asaduzzaman, et al., "Porous shaped photonic crystal fiber with strong confinement field in sensing applications: design and analysis," Sensing and Bio-Sensing Research, 2017, 13: 63-69. 